\title{
Uso do Nintendo WII como ferramenta para treinamento da cognição e equilíbrio em idosos institucionalizados
}

O envelhecimento é um processo natural, ocorrendo algumas alterações fisiológicas que irão ocorrer de forma progressiva e contínua. Uma das formas de melhora as habilidades do idoso é através de estímulo às atividades cognitivas e motoras, sendo o Nintendo uma das ferramentas que tem sido estudada, no sentido de comprovar a relação destes jogos virtuais com os aspectos equilíbrio e funcionalidade em idosos. Este trabalho teve o objetivo de avaliar o efeito da utilização do Nintendo Wii Fit nas funções cognitivas e no desempenho funcional de idosos institucionalizados. Trata-se de uma pesquisa experimental, com abordagem intervencionista quanti-qualitativa, com uma amostra de oito idosos, submetidos a avaliação cognitiva e de equilíbrio, pré e pós-intervenção, utilizando o Min Exame do Estado Mental (MEEM), o questionário Poma-Brasil. Foram submetidos a 10 intervenções com Nintendo, com 30 minutos cada. Os sinais vitais foram aferidos no início e no final de cada intervenção. Analisando os resultados do pré e pós-testes do MEEM, observa-se melhora significativa ( $p=0,05$ ); na avaliação do Poma-Brasil, houve uma melhora significativa $(p=0,02)$. O Nintendo Wii contribuiu para o treinamento da cognição e equilíbrio, mostrando-se capaz de gerar alterações hemodinâmicas positivas, contribuindo como um auxiliador no tratamento fisioterapêutico.

Palavras-chave: Idosos institucionalizados; Realidade virtual; Funcionalidade e cognição.

\section{Use of Nintendo WII as a tool for cognition and balance training in institutionalized elderly}

\begin{abstract}
Aging is a natural process, with some physiological changes occurring that will occur progressively and continuously. One of the ways to improve the skills of the elderly is by stimulating cognitive and motor activities, being Nintendo one of the tools that has been studied, in order to prove the relationship of these virtual games with balance and functionality in the elderly. This study aimed to evaluate the effect of using Nintendo Wii Fit on cognitive functions and functional performance of institutionalized elderly. This is an experimental research, with a quantitative and qualitative interventionist approach, with a sample of eight elderly, submitted to cognitive and balance assessment, pre and post intervention, using the Mini Mental State Examination (MMSE), the Poma questionnaire. Brazil. They underwent 10 interventions with Nintendo, with 30 minutes each. Vital signs were measured at the beginning and end of each intervention. Analyzing the MMSE pre and post test results, a significant improvement was observed $(p=0,05)$; In the evaluation of Poma-Brazil, there was a significant improvement $(p=0,02)$. The Nintendo Wii has contributed to the training of cognition and balance, proving to be able to generate positive hemodynamic changes, contributing as an aid in the physical therapy treatment.
\end{abstract}

Keywords: Institutionalized elderly; Virtual reality; Functionality and cognition.

Topic: Fisioterapia

Reviewed anonymously in the process of blind peer.
Received: 05/11/2018

Approved: 06/01/2019
Tamires Costa Silva

Centro Universitário Tiradentes, Brasil

http://lattes.cnpq.br/5647103136576583

tamy.cs10@gmail.com

Lauana Crysney da Silva Alves

Centro Universitário Tiradentes, Brasil

http://lattes.cnpq.br/3820227001428177

lauana.crisney@gmail.com

Rosimari de Faria Freire

Centro Universitário Tiradentes, Brasil

http://lattes.cnpq.br/9667637191341739

rosiff58@gmail.com
Referencing this:

SILVA, T. C.; ALVES, L. C. S.; FREIRE, R. F.. Uso do Nintendo WII como ferramenta para treinamento da cognição e equilíbrio em idosos institucionalizados. Scire Salutis, v.9, n.1, p.9-19, 2019. DOI: http://doi.org/10.6008/CBPC2236-9600.2019.001.0002 


\section{INTRODUÇÃO}

O envelhecimento é um processo natural, denominado senescência, período onde se pode observar algumas alterações fisiológicas, que irão ocorrer de forma progressiva e contínua (BARBOSA, 2014). A senilidade corresponde ao envelhecimento com desvio da normalidade, que pode estar acompanhada por fatores como a perda de memória e o desequilíbrio postural, que muitas vezes, levam o idoso a dependência, a introversão e ao isolamento social.

Segundo o IBGE, no período de 2000 a 2010, a população de idosos aumentou de $8,6 \%$ para $11 \%$, isto mostra que em 2010 atingimos 20 milhões de idosos, e que guardando a proporcionalidade, nas próximas duas décadas a população idosa representará $14 \%$ da população total e até 2050 , esse valor irá triplicar e no Brasil, a população de idosos, chegará aos 65 milhões (ANDRADE, 2013).

Para Rodrigues et al. (2013), a falta de apoio social para essa população é um fator que tem levado ao aumento da demanda de idosos nas Instituições de Longa Permanência (ILPIS), definida por Nunes (2010), como sendo instituições governamentais ou não, designadas a acolher pessoas com idade igual ou superior a sessenta anos e que tenham ou não o apoio familiar. Ainda segundo Rodrigues et al. (2013), a institucionalização do idoso pode ocorrer por razão de desligamento dos familiares, fragilidade na saúde do idoso e até mesmo, por conta de óbitos de entes queridos, tornando-os assim, mais propensos ao abandono.

Ferreira et al. (2010) ainda afirmou que, quanto maior o tempo de institucionalização mais fragilizado o idoso se torna, pois com a mudança do ambiente de convívio, a função cognitiva poderá ser afetada, assim como as funções psicológicas e funcionais, causando maior comprometimento à saúde e qualidade de vida do idoso, aumentando o risco para quedas, déficit na capacidade funcional, isolamento social e consequente perda de autonomia e independência nas Atividades de Vida Diária (AVD).

Fleurí (2013) concluiu em seu estudo que se torna essencial a realização de atividades lúdicas com os idosos, tendo como objetivos proporcionar momentos de lazer, bem-estar físico e psicológico, pois contribuem para a melhoria da qualidade de vida e da autonomia do idoso que se encontra institucionalizado. Nunes et al. (2010) mostrou que o uso de terapias lúdicas é um grande aliado nos tratamentos fisioterapêuticos, pois auxilia na aceitação e participação dos idosos aos exercícios propostos, fazendo com que conheçam outras formas de se exercitar, sentindo-se satisfeitos e demonstrando boa interação com o terapeuta e com os outros idosos, proporcionando bem-estar físico e psicológico bem como melhora da autonomia do idoso institucionalizado.

Uma das formas de trabalhar ludicamente com os idosos é através da realidade virtual, utilizando o Nintendo Wii, no qual possui uma plataforma virtual que contém sensores que captam os movimentos do corpo e os reproduzem na tela, em momento real, promovendo bastante interação entre o jogador e o equipamento (MUSSATO, 2012). Schiavinato et al. (2010) afirmou que se pode destacar benefícios associados a esse recurso, como a correção do equilíbrio, da postura, melhora na locomoção, na funcionalidade e melhora da função cognitiva. 
Foi demonstrado por Doná (2009) que, nas últimas décadas o uso da realidade virtual tem sido estudado por diversos autores, no sentido de se buscar comprovar a relação destes jogos virtuais interativos com os aspectos equilíbrio e funcionalidade em idosos. Já dizia Bernardes (2017), que a melhor forma de estimular e preservar algumas habilidades do idoso é através de estímulo às atividades cognitivas e motoras, fazendo com que o mesmo desenvolva e ou preserve a autonomia e segurança para a execução de atividades da vida diária, como por exemplo, tomar banho sozinho e até mesmo resolver um simples quebra cabeça com os netos. Sendo o Nintendo Wii, uma das ferramentas que abrange todos os aspectos necessários a serem trabalhados no idoso, auxiliando no tratamento fisioterapêutico.

O aumento da expectativa de vida das pessoas, o uso crescente da realidade virtual e entender as possíveis relações destes, com os aspectos funcionais e cognitivos dos idosos, justificam a escolha do tema deste trabalho. Assim, o presente estudo teve como objetivo primário avaliar o efeito da utilização do Nintendo Wii Fit, nas funções cognitivas e no desempenho funcional de idosos institucionalizados, e como objetivo secundário, avaliar o grau de comprometimento cognitivo e funcional dos idosos institucionalizados e treinar estas funções.

\section{METODOLOGIA}

A pesquisa teve caráter experimental, com abordagem intervencionista quanti-qualitativa. $\mathrm{O}$ estudo foi aprovado pelo Comitê de Ética em Pesquisa do Centro Universitário Tiradentes - UNIT/AL, sob registro 2.993.881, sendo realizado com idosos residentes em duas Instituições de Longa Permanência para Idosos (ILPIs), na cidade de Maceió (AL), eleitos de forma aleatória, para compor o $\mathrm{N}$ da pesquisa, e que atendessem aos seguintes critérios de inclusão: idosos institucionalizados, de ambos os sexos, com idade igual ou superior a 60 anos, apresentassem estabilidade hemodinâmica (pressão arterial (PA), frequência cardíaca (FC), saturação da pressão de oxigênio (SPO2) ) e aptidão cognitiva que lhes permitissem o mínimo entendimento do controle do equipamento.

Foram excluídos do estudo os idosos que apresentavam: déficit cognitivo significativo, disfunção neurológica, alterações auditivas e/ou visuais severas, os acamados, os cadeirantes, os que apresentavam dor que impossibilitavam a realização das atividades e os idosos que se negaram a participar. A Casa para Velhice Luiza de Marillac, somente abrigavam mulheres, no total de vinte e oito (28) idosas, e no Lar Evangélico Pastor Esperidião de Almeida - Abrigo LEAL, abrigavam trinta e dois (32) idosos de ambos os sexos, somando assim um total de sessenta (60) idosos.

Dos sessenta (60) idosos moradores das ILPIs, quarenta (40) foram excluídos, conforme os critérios de exclusão estabelecidos para o estudo, e apenas vinte (20) se encaixaram nos critérios de inclusão. Todos os vinte (20) idosos aprovados, foram convidados a participar da pesquisa, e a eles foram explicados todos os procedimentos que seriam realizados, e àqueles que concordaram em participar do estudo assinaram o Termo de Consentimento Livre e Esclarecido (TCLE).

A estes vinte (20) idosos selecionados, foram aplicados o questionário Mini Mental (MEEM) e o Poma-Brasil, com objetivo de conhecer os idosos que teriam condição cognitiva e equilíbrio mínimos para 
participar da intervenção com o Nitendo Wii. O Mini Exame do Estado Mental (MEEM), se trata de um instrumento, já validado, para detectar perdas cognitivas, avaliar a orientação, memória imediata, atenção e cálculo, memória de evocação e linguagem. 0 escore varia de 0 a 30, onde, quanto maior a pontuação, melhor o resultado.

Para avaliar o equilíbrio, foi utilizado o Performance Oriented Mobility Assessment (POMA), também um teste já validado, que é dividido em duas partes: uma para avaliação do equilíbrio, e a outra para avaliação da marcha, sendo permitido a aplicação de apenas uma das partes. Para este estudo foi aplicado apenas o teste de equilíbrio, que inclui avaliações de equilíbrio sentado, equilíbrio em pé, levantar-se da cadeira, entre outros. Cada questão possui um escore total bruto, que vai de: normal (3), adaptativa (2) e anormal (1). A pontuação inferior a 19, indica risco de quedas cinco vezes maior. Ou seja, quanto menor for a pontuação, maior será o problema. Foram aplicados os questionários a 20 idosos, dois (02) foram excluídos por apresentarem alteração auditiva importante que dificultava a intervenção.

Duas sessões do Nintendo Wii foram aplicadas aos 18 idosos, como forma de treinamento, familiarização ao equipamento e ao meio virtual. Alegando dificuldade em adaptação a realidade virtual, brincadeira de criança ou considerando perda de tempo, dez idosos se recusaram a permanecer no estudo, sendo, portanto, o $\mathrm{N}$ da pesquisa formado por oito idosos. A intervenção correspondeu a realização dez atendimentos, duas vezes por semana, com duração de trinta minutos cada, a todos os oito idosos. Antes e após as intervenções com o Nintendo Wii, eram colhidos os sinais vitais, que compreendiam em: aferição da pressão arterial (PA), através do esfigmomanômetro e estetoscópio da marca Premium, Frequência Cardíaca (FC) e Saturação de Oxigênio (SatO2), aferidos pelo oxímetro de pulso periférico da marca Measupro.

As intervenções ocorreram com a utilização do Nintendo Wii Fit, que possui uma plataforma (Balance Board) que capta as oscilações corporais e reproduz em ação. Os jogos utilizados foram: Pinguim Slide: participante simulado como um pinguim em cima de um bloco de gelo, em seguida no jogo é solicitado que pegue os peixes que permanecem saltando de um lado para o outro do bloco de gelo; Ski Slalom: participante simulando ser um esquiador de neve, lhe é solicitado para que passe entre as bandeiras dispostas durante o trajeto do jogo; Soccer Heading: participante simulando ser um goleiro, será orientado a rebater e cabecear as bolas e desviar-se das chuteiras e cabeças de pandas; e Table Tilt: participante simulando uma plataforma de buracos, será orientado a fazer oscilações na mesma para conseguir colocar as bolinhas que estão em cima dessa plataforma dentro dos respectivos buracos.

Os presentes jogos, foram executados com os idosos posicionados em apoio bipodal sobre a plataforma, com os objetivos de treinar ajustes posturais, estimulando o jogador a realizar oscilações corporais ântero-posterior e médio-lateral, treinando, assim, o controle de equilíbrio, lateralidade, noções espaciais e temporalidade, além disso, ampliar as habilidades para a resolução de problemas e a coordenação motora.

Os participantes, a todo tempo, estiveram assistidos e auxiliados pelas pesquisadoras durante todo o período de todas as intervenções, mantendo-se ao lado dos idosos, no sentido de prevenir os possíveis riscos de quedas. Ao final da décima sessão eles foram submetidos novamente à avaliação cognitiva e de 
equilíbrio, tendo os dados comparados. Os dados coletados foram tabulados em planilha do software EXCEL ${ }^{\circledR}$ Windows 8. Para realizar os testes estatísticos necessários foi utilizado o software BioStat 5.0, onde foram extraídos os valores de significância e as correlações existentes entre as duas amostras antes e após a aplicação com a intervenção com Nintendo Wii.

\section{RESULTADOS E DISCUSSÃO}

Entre as duas Instituições de Longa Permanência para Idosos estudadas, Casa para Velhice Luiza de Marillac e Lar Evangélico Pastor Esperidião de Almeida - Abrigo LEAL, de Maceió (AL), somaram-se sessenta idosos, sendo quarenta e três mulheres e dezessete homens. Destes, apenas vinte atenderam aos critérios de inclusão e quarenta foram excluídos por serem idosos acamados, amputados e cadeirantes. A estes vinte idosos, foram aplicados dois questionários validados, que permitiram conhecer àqueles que apresentavam mínima condição cognitiva suficiente para a utilização do Nitendo Wii, equipamento eleito para responder o questionamento do estudo, e mínimo equilíbrio suficiente para a prática do exercício em bipedestação. Dois foram excluídos por apresentarem alteração auditiva importante que dificultava a intervenção.

Após a avaliação cognitiva dos idosos, utilizando o teste Mini Exame do Estado Mental - MEEM, instrumento eleito para detectar perdas cognitivas, avaliar a orientação, memória imediata, atenção e cálculo, memória de evocação e linguagem, e também após a avaliação do equilíbrio, através do Performance Oriented Mobility Assessment (POMA), a amostra foi composta por oito indivíduos, moradores das ILPIs. Antes e após toda intervenção terapêutica, foram aferidos os sinais vitais, pressão arterial (PA), saturação da pressão de oxigênio (SpO2) e frequência cardíaca (FC).

No estudo os idosos mostraram melhora e/ou semelhança nos domínios do MEEM, quando comparados pré e pós teste, onde cinco (05) idosos ou (62,5\%), obtiveram melhora nos pós teste, e o restante, três idosos ou $(37,5 \%)$ mantiveram a mesma pontuação (tabela 1). Através da correlação de Pearson, observa-se melhora significativa $(p=0,05)$ no escore MEEM, ao analisar o pré e pós-intervenção (gráfico 1).

Tabela 1: Comparação dos domínios do MEEM no Pré e Pós-teste em Idosos Institucionalizados.

\begin{tabular}{|c|c|c|c|c|c|c|c|c|c|c|c|c|}
\hline \multirow[t]{2}{*}{ IDOSOS } & \multicolumn{2}{|c|}{ ORIENTAÇÃO } & \multicolumn{2}{|c|}{$\begin{array}{l}\text { MEMÓRIA } \\
\text { IMEDIATA }\end{array}$} & \multicolumn{2}{|c|}{$\begin{array}{l}\text { ATENÇÃO E } \\
\text { CÁLCULO }\end{array}$} & \multicolumn{2}{|c|}{$\begin{array}{l}\text { MEMÓRIA DE } \\
\text { EVOCAÇÃO }\end{array}$} & \multicolumn{2}{|c|}{ LINGUAGEM } & \multicolumn{2}{|c|}{ ESCORE } \\
\hline & PRE & PÓS & PRÉ & PÓS & & PÓS & PRÉ & PÓS & & PÓS & PRÉ & PÓS \\
\hline 1 & 2 & 8 & 3 & 3 & 0 & 5 & 0 & 1 & & 9 & 13 & 26 \\
\hline 2 & 9 & 8 & 3 & 3 & 5 & 5 & 1 & 2 & 9 & 9 & 27 & 27 \\
\hline 3 & 9 & 9 & 2 & 3 & 5 & 4 & 3 & 1 & 8 & 9 & 26 & 26 \\
\hline 4 & 10 & 10 & 3 & 3 & 5 & 5 & 2 & 2 & 9 & 9 & 29 & 29 \\
\hline 5 & 5 & 8 & 3 & 3 & 5 & 5 & 0 & 0 & 9 & 9 & 22 & 25 \\
\hline 6 & 5 & 8 & 3 & 3 & 0 & 0 & 2 & 0 & 9 & 7 & 18 & 19 \\
\hline 7 & 8 & 10 & 3 & 3 & 9 & 5 & 2 & 3 & 7 & 9 & 25 & 28 \\
\hline 8 & 9 & 10 & 3 & 3 & 5 & 5 & 2 & 3 & 7 & 7 & 25 & 27 \\
\hline
\end{tabular}

Weiss(2004) já havia citado que a reabilitação por realidade virtual tem se tornado frequente em vários Centros de Atenção à Idosos, em razão de proporcionar melhora na capacidade funcional e aumento da participação do idoso no cotidiano, o qual pode ser obtido através do treino de funções motoras, cognitivas e sensoriais, através do feedback sensório motor conquistado com o emprego do Nintendo Wii. 


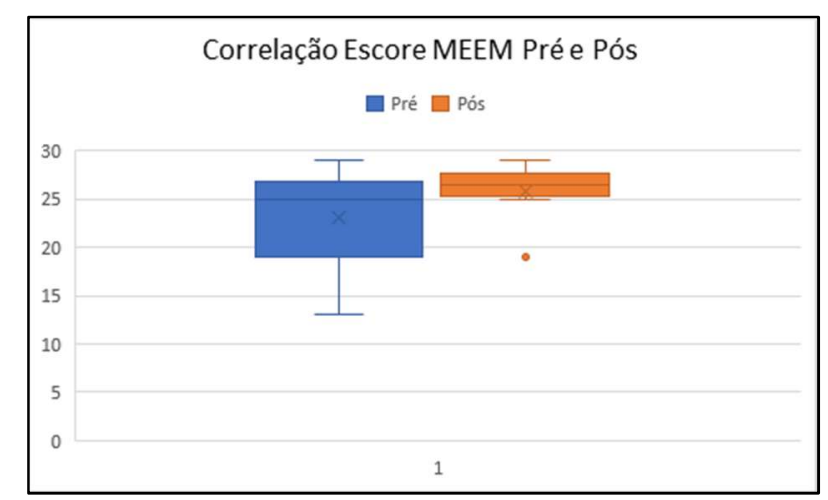

Gráfico 1: Correlação do Escore MEEM pré e pós-intervenção.

Lima (2006) mostrou que o treino cognitivo realizado de forma repetida, incide diretamente na memória, na atenção e linguagem do idoso, o que foi confirmado por Gonzaga et al. (2010), quando afirmou que quanto maior o desempenho de funções básicas, melhor será o desempenho em atividades complexas. Em um estudo realizado por Borges et al. (2008), foi avaliado a influência da realização do exercício físico, nos déficits cognitivos e funcionais de idosos frequentadores de um Centro de Saúde em Florianópolis.

Dos participantes daquele estudo, cento vinte e nove idosos, realizaram as sete avaliações apresentadas pelo MEEM, e em seguida, fizeram parte do treinamento físico por dois anos. Mostraram os autores que a média geral do MEEM apontou um aumento na pontuação, comparando-se o pré e o pós avaliação. Em relação ao treino de equilíbrio, no presente estudo, houve uma melhora significativa $(p=0,02)$ dos escores em todos os domínios do Poma-equilíbrio, comparando-se o pré e pós-intervenção (tabela 2).

Tabela 2: Comparação dos escores do POMA-BRASIL no pré e pós-teste em Idosos Institucionalizados.

\begin{tabular}{|c|c|c|}
\hline IDOSOS & PRÉ & PÓS \\
\hline $\mathbf{1}$ & 31 & 38 \\
\hline $\mathbf{2}$ & 31 & 39 \\
\hline $\mathbf{3}$ & 38 & 37 \\
\hline $\mathbf{4}$ & 36 & 39 \\
\hline $\mathbf{5}$ & 37 & 33 \\
\hline $\mathbf{6}$ & 19 & 28 \\
\hline $\mathbf{8}$ & 23 & 39 \\
\hline
\end{tabular}

Borges et al. (2017), ao avaliar as alterações fisiológicas, cognitivas e de equilíbrio com idosos de ILPI, através do Xbox terapia, participaram seis idosos, realizando dez atendimentos, três vezes por semana, com duração de 50min cada, onde os resultados não apresentaram diferença com significância estatística, mas, pode-se notar que o uso da realidade virtual influenciou positivamente sobre a funcionalidade, equilíbrio, autonomia e cognição dos idosos. No entanto, Dougherty (2011) realizou um estudo semiexperimental e intervencional, com nove idosos, com duração de $10 \mathrm{~min}$, durante três vezes na semana, por cinco semanas, indicaram que com a utilização do Wii Fit houve melhoras significativas do equilíbrio, podendo diminuir o risco de quedas.

Dos participantes do presente estudo sete idosos $(87,5 \%)$ eram hipertensos e foi observado que após a realização da atividade física, com o uso do Nintendo Wii, houve uma diferença significativa $(p=0,0005)$ na média das pressões arterial diastólica (PAD), porém, na PAS, não houve diferença significante $(p=0,81)$, pós intervenção. Observou-se a diminuição da PA nos indivíduos 1, 3, 4, 5, 6, 7, 8, pós exercício (gráfico 2). 


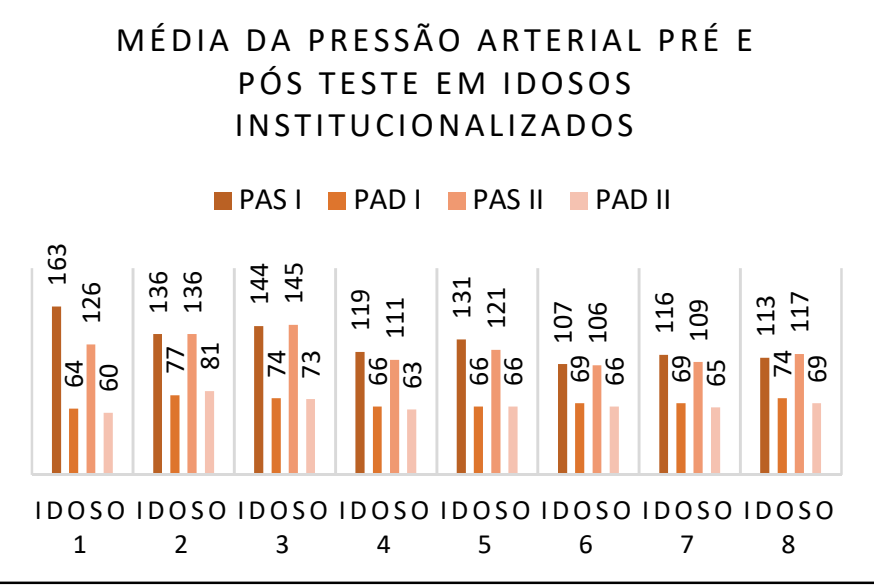

Gráfico 2: Comparação da média da pressão arterial sistólica no pré e pós-teste.

Mostarda (2009) afirmou que existem alguns fatores que alteram a pressão sanguínea, tais como: débito cardíaco, resistência periférica, elasticidade das principais artérias, viscosidade do sangue e volume sanguíneo e com o avançar da idade, a PAS irá aumentar, pelo fato das estruturas autônomas que controlam o sistema cardiovascular serem afetadas. Também, Passaro (1997), já havia afirmado que ocorre o aumento da PAS durante e após o exercício físico, sendo a elevação da PAS seguida de diminuição ou sem alteração da PA, e além disso, o aumento de PA irá depender de fatores como, postura, temperatura ambiente, duração do exercício e a interrupção súbita ou não do exercício.

MacDonald (2002) e Lizardo et al. (2005), também afirmaram em um de seus estudos que, a variação da PA ocorre de acordo com à intensidade do exercício, e por serem indivíduos idosos, a intensidade não foi elevada a ponto de ter alterações significantes causadas pelo exercício proposto. Já Fisher (2001), ao realizar um estudo com público hipertenso, disseram ter sido mais notório a hipotensão pós-exercício ao comparar com o público normotenso. Evidenciando assim, os benefícios do treinamento deste público estudado neste momento, podendo contribuir com a literatura, com mais uma opção de tratamento não medicamentoso para a hipertensão arterial.

Assim como Fisher (2001), Rondon et al. (2003) concluiu que com a prática de exercício físico regular pode haver a redução, ou até mesmo, eliminação do uso de medicamentos anti-hipertensivos. Fisher (2001) também afirmou que a queda pressórica causada pela prática de atividade física, vai depender do tempo de duração do exercício, ou seja, quanto maior o tempo, maior será a queda pressórica (45 minutos causou a queda mais rápida e acentuada, que o exercício com duração de 25 minutos).

Para Pescatello (2004), através do treinamento físico, a pressão arterial em pacientes com hipertensão pode-se reduzir significativamente. Assim como um estudo de metanálise realizado por Hagberg et al. (2000), e um estudo controlado, realizado por Whelton (2002), onde mostraram que com o treinamento físico, houve a diminuição da PAS e PAD.

Em relação a frequência cardíaca ( $F C$ ), no presente estudo utilizou somente exercícios dinâmicos, pode-se afirmar que houve uma maior resposta do débito cardíaco pelo aumento da frequência cardíaca e do volume sistólico. Havendo um aumento para os indivíduos 1, 2, 4, 6, 8, permanecendo de acordo com a literatura e não apresentando significância estatística $(p=0,09)$ (gráfico 3). 


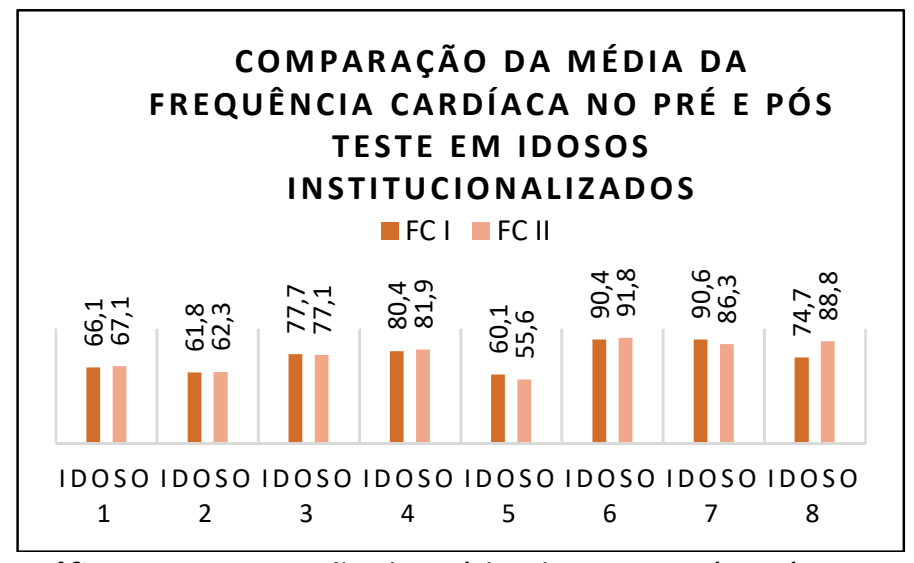

Gráfico 3: Comparação da média da FC no pré e pós-teste.

Forjaz et al. (2010) e Passaro (1997) explicaram que, durante o exercício, a pressão arterial é elevada devido ao aumento do débito cardíaco simultaneamente com a resistência vascular periférica. Em atividades físicas realizadas por idosos, observa-se que quanto maior a intensidade, maior é a FC, SpO2 e PA, onde ocorre o aumento para conseguir compensar o gasto energético, o qual está ligado ao processo de envelhecimento, onde o idoso apresenta a diminuição de força de contração no músculo cardíaco, diminuindo assim, o débito cardíaco e consequentemente a SpO2 (PETRY, 2017).

Com relação a SpO2 em si, Petry (2017), através do seu estudo, afirmou que os valores da SpO2 obtidos antes dos exercícios foram mantidos, e logo após a intervenção, os valores aumentaram, considerando assim, que as atividades relacionadas ao presente estudo não causam prejuízo na oxigenação sanguínea. Dos oito idosos estudados, quatro apresentaram elevação da $\mathrm{SpO} 2$ e os outros quatro apresentaram diminuições nestes valores, não havendo significância $(p=0,08)$ (gráfico 4).

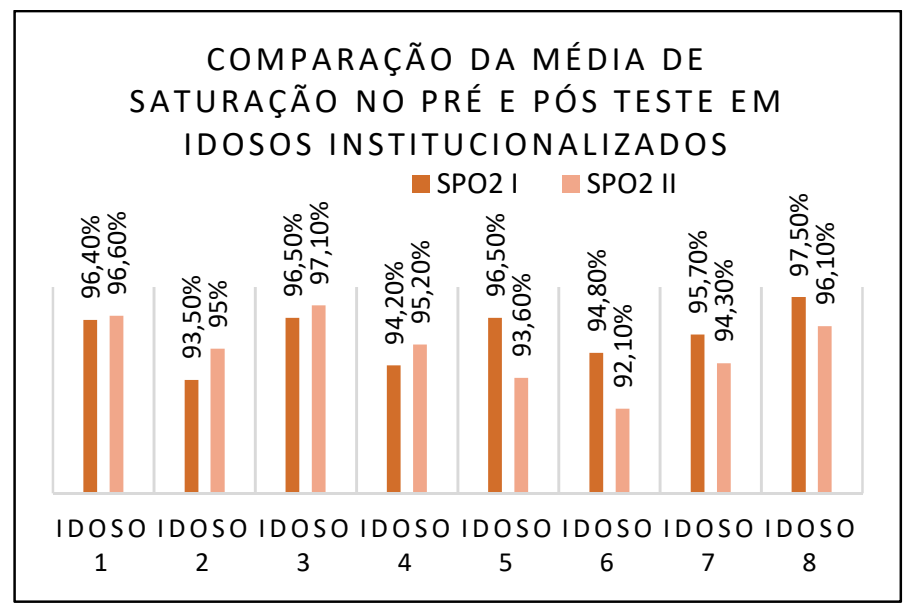

Gráfico 4: Comparação da média da SpO2 no pré e pós-teste.

O uso do Nintendo Wii é uma opção de recurso terapêutico atual, de fácil aplicação, de baixo custo, que permite a interação entre paciente e jogo, proporcionando a melhora nas capacidades sensorial e motora, e por isso, têm sido utilizado como um instrumento para a reabilitação fisioterapêutica, que auxilia na motivação do paciente em relação ao tratamento (ALBUQUERQUE et al., 2007). Carvalho (2009), em pesquisa realizada com idosos, ressaltou que os aspectos sensoriais e proprioceptivos são essenciais para o aperfeiçoamento e melhora do equilíbrio e propriocepção. 
Segundo Ergen et al. (2008), os programas de exercícios devem ser realizados de forma regular, contínua e gradual, tanto em aspectos de intensidade, quanto de complexidade. Porém, no presente estudo observou-se que, por conta das fases dos jogos do Nintendo Wii não possuírem continuidade, o fisioterapeuta terá sempre que reiniciar as fases do jogo, para alcançar um período de duração e intensidade suficientes para o aprendizado motor e proprioceptivo. Estes aspectos podem e deveriam ser melhorados, tornando-o equipamento mais efetivo e específico para a reabilitação fisioterapêutica.

Para Treml (2013), a vontade do indivíduo em executar bem as jogadas pode provocar efeitos positivos no organismo como, facilidade para recuperação dos movimentos, estímulo da atividade cerebral, fortalecimento muscular, aumento da capacidade de concentração e equilíbrio. Segundo Ergen et al. (2008) realizaram um estudo com trinta e dois idosos, durante dez atendimentos, duas vezes na semana, com duração de 30min, sendo que dividiram em grupo controle (treino de propriocepção e equilíbrio convencional) e grupo experimental (treinamento proprioceptivo e uso do Nintendo Wii), e concluíram que o treinamento proprioceptivo através do Nintendo Wii foi mais eficaz que o treinamento proprioceptivo convencional, em relação ao equilíbrio, mobilidade, flexibilidade e quedas em indivíduos idosos.

Gómez (2011) também afirmou a efetividade da reabilitação com o Nintendo Wii, quando comparado a um recurso convencional. A tabela 3 apresenta que entre os idosos que participaram do estudo havia sete que realizavam fisioterapia, destes, quatro realizavam fisioterapia duas vezes na semana (idosos $2,3,4,5$ ), e três idosos (Idosos 6, 7, 8) realizavam fisioterapia quatro vezes na semana, sendo que em dois (02) dias, faziam fisioterapia nos dois horários. Apenas um participante da pesquisa não realizava fisioterapia.

Tabela 3: Número de Idosos que realizavam fisioterapia concomitante ao estudo.

\begin{tabular}{|c|c|c|c|}
\hline IDOSOS & FISIOTERAPIA & SEMANA & FREQUÊNCIA \\
\hline $\mathbf{1}$ & -- & -- & -- \\
\hline $\mathbf{2}$ & Realiza & $2 \mathrm{X}$ & 1 Turno \\
\hline $\mathbf{3}$ & Realiza & $2 \mathrm{X}$ & 1 Turno \\
\hline $\mathbf{4}$ & Realiza & $2 \mathrm{X}$ & 1 Turno \\
\hline $\mathbf{5}$ & Realiza & $2 \mathrm{X}$ & 2 Turnos \\
\hline $\mathbf{6}$ & Realiza & $4 \mathrm{X}$ & 2 Turnos \\
\hline $\mathbf{7}$ & Realiza & $4 \mathrm{X}$ & 2 Turnos \\
\hline $\mathbf{8}$ & Realiza & $4 \mathrm{X}$ & \\
\hline
\end{tabular}

Com base nos resultados obtidos, e em razão dos idosos que participaram do estudo estarem realizando fisioterapia convencional concomitante às atividades com o Nintendo Wii, não foi possível afirmar que o equipamento, por si só, contribuiu para o treinamento da cognição e equilíbrio, $n$ entanto, mostrouse ser capaz de gerar alterações positivas hemodinâmicas, alterações no equilíbrio, entre outras alterações.

\section{CONSIDERAÇÕES FINAIS}

Foi possível observar que, o Nintendo Wii, concomitante às atividades da fisioterapia convencional, contribuiu para o treinamento da cognição e equilíbrio, mostrando-se capaz de gerar alterações positivas hemodinâmicas e alterações no equilíbrio, o que é eficaz na prevenção de quedas, incrementa a melhora da mobilidade, da flexibilidade e influencia na capacidade de concentração e memória. 
Sendo assim, conclui-se que o Nintendo Wii, como recurso fisioterapêutico, é um grande aliado para a manutenção da performance dos idosos, que se encontra comprometida em decorrência aos fatores da senilidade, podendo assim, o equipamento contribuir no processo de reabilitação, sempre associado ao tratamento convencional e não como um único recurso. Sugerem-se novos estudos, tendo um grupo controle com o Nitendo Wii, para que se possa conhecer os efeitos deste equipamento como recurso terapêutico individual, e afirmar como um possível recurso para a incrementação do cognitivo e equilíbrio de idosos.

\section{REFERÊNCIAS}

ALBUQUERQUE, E. C.; SCALABRIN, E. E.. O uso de computador em programas de reabilitação neuropsicológico. Psicologia Argumento, Curitiba v.25, n.50, p.267-273, 2007.

ANDRADE, L.. Políticas Públicas para Pessoas Idosas no Brasil: Uma Revisão Integrativa. Ciência \& Saúde Coletiva, Rio de Janeiro, v.18, n.12, p.3543-3552, 2013.

BARBOSA, B. R.. Avaliação da Capacidade Funcional dos Idosos e Fatores Associados a Incapacidade. Ciência \& Saúde Coletiva, v.19, p.3317-3325, Rio de Janeiro, 2014.

BERNARDES, F. R.. Envelhecimento e Queixa Subjetiva de Memória e a Relação com a Fluência Verbal em Idosos Ativos. CoDAS, São Paulo, v.29, n.3, 2017.

BORGES, A. P. M.; MENDES, G. C.. Avaliação cognitiva e de equilíbrio em idosos institucionalizados após intervenção de xbox terapia. Cadernos da Escola de Saúde, Curitiba, v.1, n.13, 2017.

BORGES, L. J.; BENEDETTI, T. R. B.; MAZO, G. Z.. Exercício físico, déficits cognitivos e aptidão funcional de idosos usuários dos centros de saúde de Florianópolis. Revista Brasileira de Atividade Física \& Saúde, Florianópolis, v.13, n.3, p.167-177, 2008.

CARVALHO, S. S.. Análise do equilíbrio dos idosos através da biofotogrametria computadorizada pós treinamento proprioceptivo. In: ENCONTRO LATINO DE INICIAÇÃO CIENTÍFICA, IX ENCONTRO LATINO AMERICANO DE PÓSGRADUAÇÃO E III ENCONTRO LATINO DE INICIAÇÃO CIENTÍFICA JUNIOR, 13. Anais. São José dos Campos: Universidade do Vale do Paraíba, 2009.

DONÁ, F.. Uma Abordagem Interdisciplinar na Avaliação e Reabilitação do Idoso com Disfunção Vestibular Crônica. Revista Equilíbrio Corporal e Saúde, v.1, n.1, p.2232, São Paulo, 2009.

DOUGHERTY, J.. The effects of a multi-axis balance board intervention program in an elderly population. Missouri Medicine, Missouri, v.108, n.2, p.128, 2011.

ERGEN, E.; ULKAR, B.. Proprioception and ankle injuries in soccer. Clinics in Sports Medicine, Ancara, v.27, n.1, p.195217, 2008.

FERREIRA, D. C. O.; YOSHITOME, A. Y.. Prevalência e Caraterísticas das Quedas de Idosos Institucionalizados. Revista Brasileira de Enfermagem, São Paulo, v.63, n.6, p.991-997, 2010.
FISHER, M. M.. The effect of resistance on recovery blood pressure in normotensive and boderline hypertensive women. The Journal of Strenght Conditioning Research, v.15, n.2, p.120-126, 2001.

FLEURÍ, A. C. P.. Atividades Lúdicas com Idosos Institucionalizados. Revista de Enfermagem, v.16, n.1, p.50$57,2013$.

FORJAZ, C. L. M.; QUEIROZ, A. C. C.; CARDOSO JÚNIOR, C. G.. Exercício físico na hipertensão arterial: riscos e benefícios. In: BENTO, J. O.; TANI, G.; PRISMA, A.. Desporto e educação física em português. Porto: 2010. p. 256-273.

GONZAGA, L.; NUNES, B.. Memória Funcionamento Perturbações e Treino. Lisboa: 2008.

HAGBERG, J. M.; PARK, J. J.; BROWN, M. D.. The role of exercise training in the treatment of hypertension na update. Sports Medicine, v.30, p.193-206, 2000.

LIMA, J.. Envelhecimento, demência e doença de Alzheimer: o que a psicologia tem a ver com isso?. Revista de Ciências Humanas, Florianópolis, v.40, p.469-489, 2006.

LIZARDO, S. H. F; SIMÕES, H. G.. Efeitos de diferentes sessões de exercícios resistidos sobre a hipotensão pós exercício. Revista Brasileira de Fisioterapia, v.9, n.3, 2005.

MACDONALD, J. R.. Potential causes, mechanisms and implications of post exercise hypotension. Journal of Human Hypertension, v.16, n.4. p.225-236, 2002.

MOSTARDA, C.. Hipertensão e modulação autonômica no idoso: papel do exercício físico. Revista Brasileira de Hipertensão, v.16, n.1, p.55-60, 2009.

MUSSATO, R.; BRANDALIZE, D.; BRANDALIZE, M.. Nintendo Wii e seu efeito no equilíbrio e marcha de idosos saudáveis. Revista Brasileira de Ciência e Movimento, Brasília, v.20, n.2, p.68-75, 2012.

NUNES, V. M. A.; MENEZES, R. M. P.; ALCHIERI, J.

C.. Avaliação da Qualidade de Vida em Idosos Institucionalizados no Município de Natal, Estado do Rio Grande do Norte. Acta Scientiarum: Health Sciences, v.32, n.2, p.119-126, 2010.

PASSARO, L. C.. Resposta cardiovascular na prova de esforço: pressão arterial sistólica. Revista Brasileira de Medicina do Esporte, v.3, n.1, p.6-10, 1997. 
PESCATELLO, L. S.. Exercise and hypertension. Medicine \& Science in Sports \& Exercise, v.36, n.3, p.533-553, 2004.

PETRY, A. L. N. C.. Influência da atividade física em grupo na qualidade de vida e variáveis cardiorrespiratórias em idosos institucionalizados. Revista FisiSenectus, Chapecó, v.4, n.1, 2017.

RODRIGUES, A. G.; SILVA, A. A.. A Rede Social e os Tipos de Apoio Recebidos por Idosos Institucionalizados. Revista Brasileira de Geriatria e Gerontologia, Rio de Janeiro, v.16, n.1, p.159-170, 2013.

RONDON, M. U. P. B.; BRUM, P. C.. Exercício físico como tratamento não farmacológico da hipertensão arterial. Revista Brasileira de Hipertensão, São Paulo, v.10, p.134137, 2003.
SCHIAVINATO, A. M.; BALDAN, C.; MELATTO, L.; LIMA, L. S. L.. Influência do Wii Fit no Equilíbrio de Paciente com Disfunção Cerebelar: Estudo de Caso. Journal of the Health Science Institute, São Paulo, v.28, n.1, p.50-52, 2010.

TREML, C. J.. O Uso da plataforma Balance Board como Recurso Fisioterápico em Idosos. Revista Brasileira de Geriatria e Gerontologia, Rio de Janeiro, v.16, n.4, p.759768, 2013.

WEISS, P. L. W.. Video capture virtual reality as a flexible and effective rehabilitation tool. Journal of Neuro Engineering and Rehabilitation, v.1, n.1, Haifa, p.1-12, 2004.

WHELTON, S. P.. Effect of aerobic exercise on blood pressure: a meta-analysis of randomized, controlled trials. Annals of Internal Medicine, v.136, n.7, p.493-503, 2002.

A CBPC - Companhia Brasileira de Produção Científica (CNPJ: 11.221.422/0001-03) detém os direitos materiais desta publicação. Os direitos referem-se à publicação do trabalho em qualquer parte do mundo, incluindo os direitos às renovações, expansões e disseminações da contribuição, bem como outros direitos subsidiários. Todos os trabalhos publicados eletronicamente poderão posteriormente ser publicados em coletâneas impressas sob coordenação da Sustenere Publishing, da Companhia Brasileira de Produção Científica e seus parceiros autorizados. Os (as) autores (as) preservam os direitos autorais, mas não têm permissão para a publicação da contribuição em outro meio, impresso ou digital, em português ou em tradução. 